\title{
The Use of Hypothermically Stored Amniotic Membrane for Cartilage Repair: A Sheep Study
}

\author{
Samuel K. Tabet' ${ }^{1}$ Alisa L. Clark ${ }^{2}$, Eric B. Chapman', Doug Thal ${ }^{3}$ \\ ${ }^{1}$ New Mexico Orthopaedic Associates, Albuquerque, NM, USA \\ ${ }^{2}$ School of Medicine, University of New Mexico, Albuquerque, NM, USA \\ ${ }^{3}$ Thal Equine LLC, Albuquerque, NM, USA \\ Email: TabetSK@nmortho.net, alisaclark77@gmail.com, chapmaneb@nmortho.net, \\ drthal@thalequine.com
}

Received 15 September 2015; accepted 26 October 2015; published 30 October 2015

Copyright (C) 2015 by authors and Scientific Research Publishing Inc.

This work is licensed under the Creative Commons Attribution International License (CC BY). http://creativecommons.org/licenses/by/4.0/

(c) (i) Open Access

\section{Abstract}

Objective: To evaluate the use of hypothermically stored human amniotic membrane for cartilage repair in adult sheep. Studies show that human amniotic membrane contains pluripotent mesenchymal stem cells that can be influenced to produce chondrocytes. It is unknown if human amniotic cells can produce hyaline-like cartilage. This study evaluates the use of hypothermically stored amniotic membrane (HSAM) to fill chondral defects in a sheep model. We hypothesized HSAM would fill defects with hyaline-like cartilage with chondrocytes populating the matrix. One sheep was used as a control, and four sheep received amniotic membrane. Two of these sheep were used as a normal control comparison. A $1 \mathrm{~cm}^{2}$ defect was created on the trochlear grove in all specimens. Each membrane was sized and laid over with the stromal layer facing the subchondral bone and covered with Fibrin sealant. The knees were harvested at five months and underwent morphological, histological, and immunohistological evaluation based on the original validated scoring system by 0 'Driscoll. The control defect didn't fill in with hyaline cartilage or fibrocartilage. The defects that successfully retained the graft had evidence of diffuse chondrocyte cell proliferation and showed a stromal matrix similar to hyaline cartilage. The graft samples showed a near $100 \%$ morphological fill in the HSAM defect contrasting to $<10 \%$ fill in the control defect. The retained HSAM grafts scored 2.5 on a 0 - 3 cartilage appearance scale compared with 0.5 for the control defects. HSAM is a potential source of pluripotent cells that can influence chondrogenesis in a sheep model. The implications for application in a human model are promising and warrant further study.

\footnotetext{
*Corresponding author.
} 


\section{Keywords}

\section{Mesenchymal Stem Cells, Chondrocytes, Cartilage Repair, Amniotic Membrane}

\section{Introduction}

Addressing articular cartilage defects is challenging, especially when the defect is large $\left(>2.0-2.5 \mathrm{~cm}^{2}\right)$. This is due in large part to avascularity and the limited self-regeneration nature of articular cartilage [1]. There are various methods to address cartilage defects, each with the goal of producing fibro-cartilage or hyaline-like cartilage [2]. However, each method has associated drawbacks. Microfracture consists of drilling into the subchondral bone to stimulate the marrow. Microfracture produces fibrocartilage but is not recommended for large lesions $\left(>2.5 \mathrm{~cm}^{2}\right)$ and the longevity of the repair is questionable. Alternatively, there are options available that allow for the filling of the defect with hyaline-like cartilage with chondrocytes in the stromal matrix of variable sources, both of autograft and allograft origin [3]. Autologous Chondrocyte Implantation (ACI) allows for the filling of the defect with hyaline-like cartilage but often results in hypertrophy and adhesions, leading to a minimum of two to three procedures, and it is expensive. Autologous Osteochondral Transplantation (OATS), is an osteochondral autograft, but is limited to treating small chondral defects, is technically difficult and can cause damage to the nearby articular cartilage, resulting in a significant morbidity from the donor sites. DeNovo NT is a single procedure, is less expensive and the implantation procedure is simple, however, product availability can be an issue. Finally, there are many other less known investigational cellular treatments in Europe and other countries which are too numerous to list. A product that is readily available, technically easy, inexpensive and induces minimal morbidity to the patient would be ideal for cartilage restoration and repair [3].

Recent studies have demonstrated the availability of pluripotent cells from fresh amniotic membrane (AM) with the ability to grow hyaline-like cartilage and fill cartilage defects [3]-[8]. It has been shown that amniotic cells contain pluripotent mesenchymal stem cells that can be influenced to produce various cell lines including chondrocytes and contain important growth factors, such as proteoglycans (i.e. biglycan, decorin, and perlecan) and glycosamineoglycans [3] [6]-[8]. In vitro studies involving both rabbit and human chondrocytes have also demonstrated the pluripotent nature of AM [9]. AM also contains a variety of anti-inflammatory factors including interleukin 10 (IL-10) and interleukin 1 receptor antagonist (IL-1ra) [10].

Sterilely procured amniotic membrane has only begun to be explored but has the advantage of being readily available as compared to other allogeneic sources of pluripotent cells. The application of this as a treatment for chondral defects becomes less technically demanding. This is especially important when combining this technique with other procedures such as ligament reconstruction, meniscal transplant, hightibial osteotomy and/or patella realignment. This study evaluates the use of hypothermically stored amniotic membrane (HSAM) [1] to fill cartilage defects in a sheep model. It was hypothesized that HSAM would be able to fill cartilage defects with cell proliferation of a stromal matrix similar to hyaline cartilage. Cartilage growth was evaluated morphologically, histologically, and immunohistologically.

\section{Methods}

\subsection{Experimental Design and Surgery}

Five adult sheep, each less than three years old, were chosen for the study. The sheep were purchased from a local sheep dealer and were all healthy Suffolk-cross ewes. In accordance with the local Ethical Committee, all of the sheep were evaluated by a licensed veterinarian and determined to be healthy without any limb deformity prior to this study.

Preoperatively, a licensed veterinarian administered to each sheep 1 gram of phenylbutazone, $10 \mathrm{ml}$ procaine penicillin G IM, and 2 - $75 \mathrm{mcg}$ fentanyl patches applied to the forearm. $3.3 \mathrm{mg} / \mathrm{kg}$ of ketamine combined with $0.3 \mathrm{mg} \cdot \mathrm{kg}$ of midazolam was given intravenously to anesthetize the sheep. The sheep were maintained on IPPV and inhaled isofluorane. In addition, a CRI of ketamine $(10 \mathrm{mcg} / \mathrm{kg} / \mathrm{min})$ and lidocaine $(25 \mathrm{mcg} / \mathrm{kg} / \mathrm{min}$ diluted in LRS) was administered during anesthesia. Each sheep was also administered 1 gram of cefazolin at the start of anesthesia and 30 minutes following anesthesia. Penicillin and phenylbutazone were maintained four days post operatively. The fentanyl patches were removed at post-operative day three. The hindquarter knee of each 
sheep was sterilized and surgically exposed. A median parapatellar incision was made by a single surgeon on each of the five sheep. A $1 \mathrm{~cm}^{2}$ chondral defect was created on the trochlea of each sheep using a cuvette without penetration of the subchondral bone. An attempt was made to spare the calcified cartilage layer. Immediately following the creation of the lesion, four sheep received the HSAM graft. Affinity is a prepackaged and sterilely procured amniotic membrane stored in a normal saline solution in a hypothermal environment (versus cryopreserved). It was used in a bilayer technique to fill the cartilage defect. Care was taken to place the epithelial layer away from the bone defect, as this layer does not have pluripotent cells. The membrane was folded so that the pluripotent mesenchymal cellular layer faced the defect and the joint. The membrane was fixed to the trochlear defects using Tisseal fibrin glue.

The fifth sheep was used as a defect control, and was not filled in with HSAM. Wounds were closed in a standard fashion with non-absorbable sutures in the knee capsule. The skin and dressings were a topical silver adhesive. The wounds were closed and the sheep were allowed to bear weight as tolerated without immobilization immediately after surgery. Following harvesting, normal cartilage was removed from two of the four sheep from the opposite condyle and used as normal control comparisons in the histological and immunohistochemistry evaluation. The final sample included four HSAM grafts, two normal control comparisons, and one defect control comparison.

\subsection{Examination of the Defects}

Sheep were evaluated by a licensed large animal veterinarian at two weeks, six weeks, and five months. Wounds were assessed for infection and swelling and joint effusion, lameness and ROM were evaluated. An in-house lameness scale was used to evaluate lameness. This scale ranges from $0-5$, with $0=$ "no perceptible lameness," and 5 = "lameness produces minimal weight bearing in motion and/or at rest or a complete inability to move". ROM was evaluated by comparison of the operative knee to the non-operative knee. At five months postprocedure, the sheep were sacrificed and the distal femurs were harvested. Histological samples were taken of the operative sites, both those treated with HSAM and those not treated with HSAM. Normal cartilage samples were taken from two of the implanted sheep for comparison using a $2.0 \mathrm{~mm}$ Miltexcoring instrument to minimize cartilage damage. Cross morphologic evaluations were performed and the same coring instruments were used to obtain histological samples from the implanted specimens. Samples were placed in a standard solution of formalin and taken to the lab at the University of Alabama for histological preparation.

The samples were stained with Hemotoxylin and Eosin ( $\mathrm{H} \& \mathrm{E}$ ), Safranin-O, and Masson's Trichrome. The original validated cartilage scoring system developed by O’Driscoll was used to compare cartilage growth in the H \& E stains of the HSAM and control samples [11]. This evaluation method rates cartilage growth on two levels. First, the sample is rated 0 - 3 on overall appearance $(0=$ no cartilage present, $3=$ mostly normal appearing cartilage), which was referred to as "simple score.” Second, each sample was given a percent (\%) value of cartilage-like tissue; these values were referred to as “\% score.” All samples were evaluated by three independent raters and compared to a normal sample of cartilage. The raters were blinded to the results of the other raters.

\subsection{Analysis}

Joint effusion, lameness and ROM were evaluated at two weeks, six weeks, and five months post operatively. Cartilage growth was evaluated by three independent raters both morphologically and histologically using the O'Driscoll cartilage scoring method. The reliability of the histological cartilage ratings were evaluated using Predictive analytics software and solutions (SPSS) statistical software. The relationship between graft type, simple and percent scores were evaluated using the Pearson chi square test. The relationship between the individual raters and simple and percent scores were evaluated using the Freeman Halton fisher exact test and Wilcoxon rank sum test. Safranin-O stains were taken to evaluate the presence of proteoglycan productivity and Masson's Trichrome stains were taken to evaluate integration of the base of the cartilage to the subchondral bone. Immunohistochemistry was performed in order to evaluate the presence of Type II collagen in the treated grafts compared to control and normal grafts.

\section{Results}

When evaluated prior to sacrifice, the sheep indicated recovery from the procedure. Upon gross evaluation, it 
was found that $50 \%$ of the HSAM grafts retained the membrane grossly. Two of the grafts were presumably dislodged, indicated by a close resemblance to the control defect. For this reason, analysis of the samples was performed only on those samples that retained the amniotic membrane. All sheep, excluding the HSAM grafts that were not retained, were rated at a lameness level of 1 - 2 (lameness difficult to observe or is not perceptible) at final evaluation and had only mild-moderate effusion. Full ROM was established at five months post operation (Table 1). In both the control and experimental defects no features of inflammation, hypertrophy or ossification were observed.

Upon evaluation, the graft samples showed a near $100 \%$ morphological fill in the HSAM defect contrasting to less than a $10 \%$ fill in the control defect. The chondral defects created on the femoral surface did not create lesions on the tibial side. The two experimental defects that retained their membranes had evidence of diffuse chondrocyte-like cell proliferation and showed a stromal matrix that included hyaline cartilage. $\mathrm{H} \& \mathrm{E}$ staining showed that the HSAM graft samples had evidence of unorganized hypercellular infiltrate of chondrocytes in the lacunae, extending all the way to the articular surface with a significant integration to the surrounding host cartilage. Also present was a fibrous component of the stromal matrix thereby indicating the presence of mixed hyaline cartilage. At the base of the lesion, there was a reestablishment of the calcified cartilage layer (Figures 1-3). The retained grafts all scored 2.5 on a 0 - 3 cartilage appearance scale compared with 0.5 for the controls (Table 2). Graft type was related to both simple score and percent score, as expected ( $\chi^{2}=1, p=0.007$ for both) (Table 2, Table 3). There was no difference in simple or percent scores among the three independent raters

Table 1. Lameness, effusion, ROM.

\begin{tabular}{|c|c|c|c|c|}
\hline Sheep & Evaluation & Lameness (0 - 5) & Effusion & ROM \\
\hline \multirow[t]{3}{*}{ Sheep 28 (Normal) } & 2 wks & 2 & Moderate & \\
\hline & 6 wks & 2 & Mild & \\
\hline & $5 \mathrm{mo}$ & $2-$ & Mild & full, $\mathrm{nml}$ \\
\hline \multirow[t]{3}{*}{ Sheep 28 (HSAM) } & 2 wks & - & - & \\
\hline & 6 wks & - & - & \\
\hline & $5 \mathrm{mo}$ & - & - & - \\
\hline \multirow[t]{3}{*}{ Sheep 43 (Control) } & 2 wks & $1+$ & Mild & \\
\hline & 6 wks & $1+$ & Mild & \\
\hline & $5 \mathrm{mo}$ & $1+$ & Mild & full, nml \\
\hline \multirow[t]{3}{*}{ Sheep 56 (HSAM) } & 2 wks & - & - & \\
\hline & 6 wks & - & - & \\
\hline & $5 \mathrm{mo}$ & - & - & - \\
\hline \multirow[t]{3}{*}{ Sheep 72 (HSAM) } & 2 wks & $2+$ & Moderate & \\
\hline & 6 wks & $2+$ & Mild & \\
\hline & $5 \mathrm{mo}$ & $2-$ & Mild & full, nml \\
\hline \multirow[t]{3}{*}{ Sheep 82 (Normal) } & 2 wks & $2+$ & Mild & \\
\hline & 6 wks & 2 & Mild & \\
\hline & $5 \mathrm{mo}$ & 2 & Mild & full, nml \\
\hline \multirow[t]{3}{*}{ Sheep 82 (HSAM) } & 2 wks & 2 & Moderate & \\
\hline & 6 wks & $2+$ & Mild & \\
\hline & $5 \mathrm{mo}$ & $2+$ & Mild & full, nml \\
\hline
\end{tabular}

After five months, all sheep were not lame and showed only mild to moderate effusion. 2/4 HSAM grafts were not retained (Sheep 28 and Sheep 56). Sheep are labeled above as they were labeled during the experiment. 


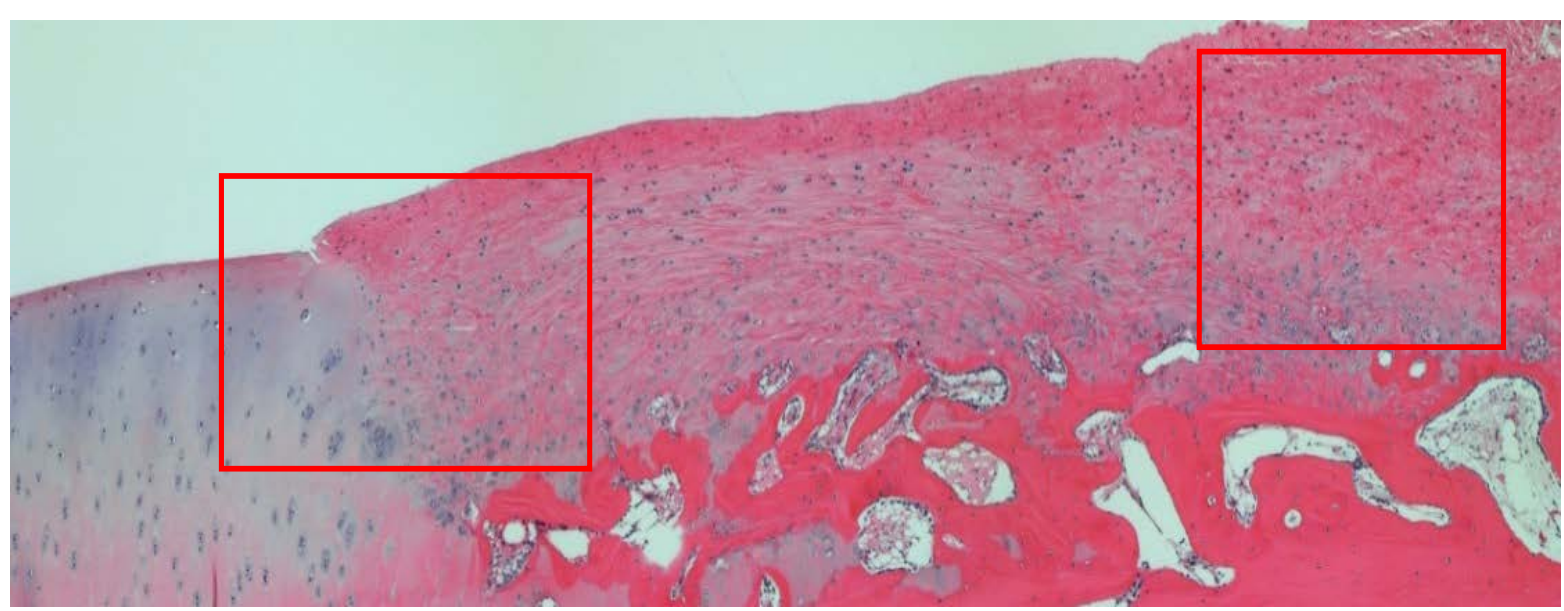

(a)

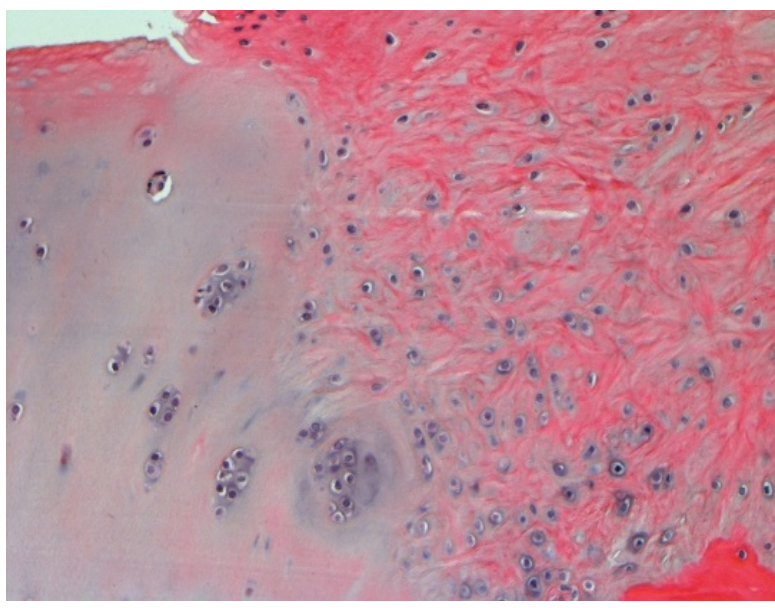

(b)

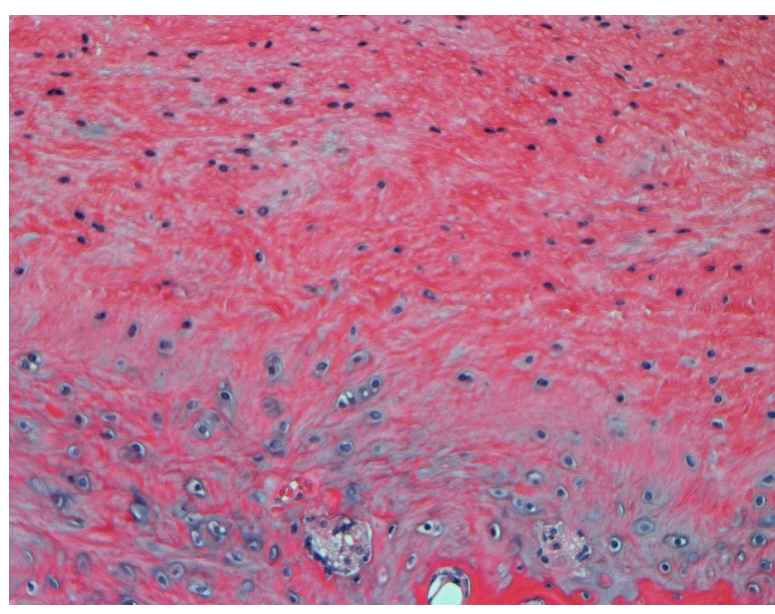

(c)

Figure 1. (a) 10X Magnification, H \& E stain of a HSAM sample of cartilage. (b) 20X Magnification, H \& E stain of a HSAM sample of cartilage. Margin integration of new cartilage to existing normal cartilage. (c) 20X Magnification, H \& E stain of a HSAM sample of cartilage. Integration to subchondral bone and proliferation of chondrocytes in lacunae at the base of the defect.



(a)

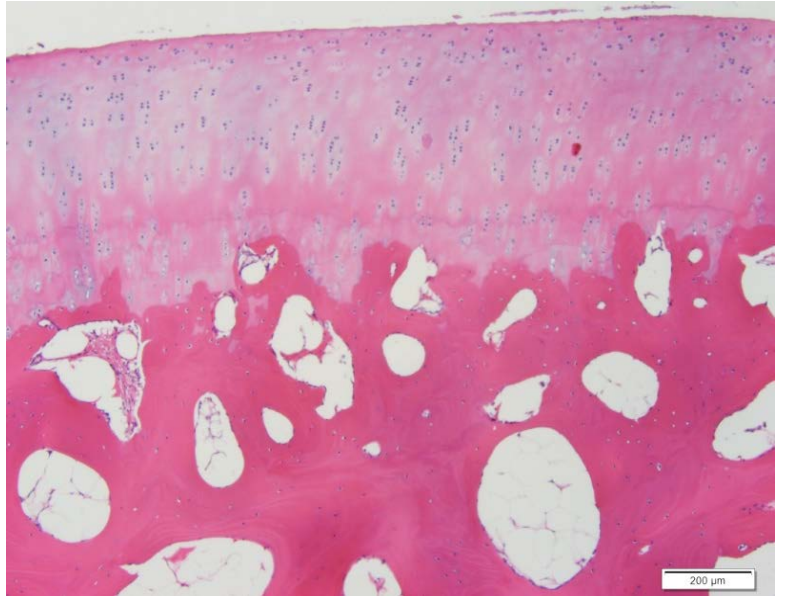

(b)

Figure 2. (a) 4X Magnification, H \& E stain of normal cartilage. (b) 10X Magnification, H \& E stain of normal cartilage. 


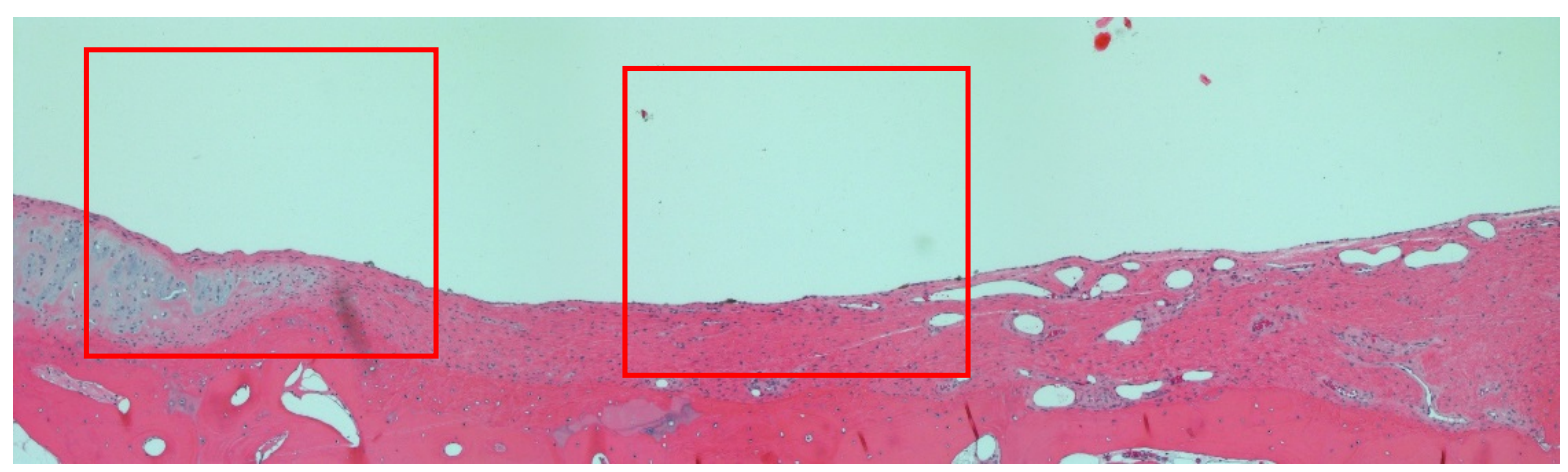

(a)



(b)

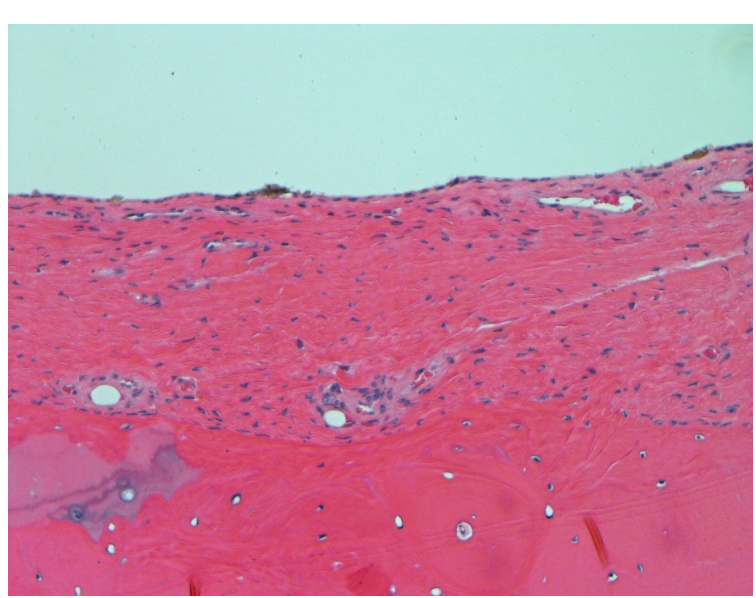

(c)

Figure 3. (a) 10X Magnification, $\mathrm{H} \& \mathrm{E}$ stain of a control sample of cartilage. No new cartilage integration at the margin in the control sample. (b) 20X Magnification, H \& E stain of a control sample. (c) 20X Magnification, H \& E stain of a control sample. No new cartilage integration at the margin in the control sample.

Table 2. Summary of graft type by simple score.

\begin{tabular}{cccccccccc}
\hline & \multicolumn{9}{c}{ Simple Score } \\
\cline { 2 - 9 } Graft Type & \multicolumn{3}{c}{ Rater 1 } & \multicolumn{3}{c}{ Rater 2 } & \multicolumn{3}{c}{ Rater 3 } \\
\cline { 2 - 9 } & $\mathbf{0 . 5}$ & $\mathbf{2 . 5}$ & $\mathbf{3 . 0}$ & $\mathbf{0 . 5}$ & $\mathbf{2 . 5}$ & $\mathbf{3 . 0}$ & $\mathbf{0 . 5}$ & $\mathbf{2 . 5}$ & $\mathbf{3 . 0}$ \\
\hline Control & $1(100 \%)$ & $0(0 \%)$ & $0(0 \%)$ & $1(100 \%)$ & $0(0 \%)$ & $0(0 \%)$ & $1(100 \%)$ & $0(0 \%)$ & $0(0 \%)$ \\
HSAM & $0(0 \%)$ & $2(100 \%)$ & $0(0 \%)$ & $0(0 \%)$ & $2(100 \%)$ & $0(0 \%)$ & $0(0 \%)$ & $2(100 \%)$ & $0(0 \%)$ \\
Normal & $0(0 \%)$ & $0(0 \%)$ & $2(100 \%)$ & $0(0 \%)$ & $0(0 \%)$ & $2(100 \%)$ & $0(0 \%)$ & $0(100 \%)$ & $2(100 \%)$ \\
\hline
\end{tabular}

$\left(\chi^{2}=1, \mathrm{p}=0.007\right)$.

Table 3. Summary of graft type by \% score.

\begin{tabular}{cccccccccc}
\hline & \multicolumn{9}{c}{ \% Score } \\
\cline { 2 - 9 } Graft Type & \multicolumn{3}{c}{ Rater 1 } & \multicolumn{3}{c}{ Rater 2 } & \multicolumn{3}{c}{ Rater 3} \\
\cline { 2 - 9 } & $\mathbf{1 6 . 6 0}$ & $\mathbf{8 3 . 3 3}$ & $\mathbf{1 0 0 . 0 0}$ & $\mathbf{1 6 . 6 0}$ & $\mathbf{8 3 . 3 3}$ & $\mathbf{1 0 0 . 0 0}$ & $\mathbf{1 6 . 6 0}$ & $\mathbf{8 3 . 3 3}$ & $\mathbf{1 0 0 . 0 0}$ \\
\hline Control & $1(100 \%)$ & $0(0 \%)$ & $0(0 \%)$ & $1(100 \%)$ & $0(0 \%)$ & $0(0 \%)$ & $1(100 \%)$ & $0(0 \%)$ & $0(0 \%)$ \\
HSAM & $0(0 \%)$ & $2(100 \%)$ & $0(0 \%)$ & $0(0 \%)$ & $2(100 \%)$ & $0(0 \%)$ & $0(0 \%)$ & $2(100 \%)$ & $0(0 \%)$ \\
Normal & $0(0 \%)$ & $0(0 \%)$ & $2(100 \%)$ & $0(0 \%)$ & $0(0 \%)$ & $2(100 \%)$ & $0(0 \%)$ & $0(100 \%)$ & $2(100 \%)$ \\
\hline
\end{tabular}

$\left(\chi^{2}=1, \mathrm{p}=0.007\right)$. 
(Table 4). Safranin-O stains (Figure 4) indicated the presence of proteoglycan productivity and Masson's Trichrome stain (Figure 5) showed significant integration of the cartilage base with thesubchondral bone. Immunohistochemistry exhibited Type II collagen at the base of both successful affinity patches (Figure 6). Type II Collagen was not visualized in the control grafts (Figure 7).

\section{Discussion}

There are multiple advantages of using a sterilely procured amniotic membrane for cartilage restoration. HSAM has the advantage of being more readily available as compared to other allogenic sources of live pluripotent cells. In addition, HSAM is less likely to endure tissue structural damage due to it being a live tissue and the way it is preserved. Further, this single technique has advantages over more complicated procedures involving suturing membranes. The one step application suggests a less expensive alternative in a world seeking efficient and economical medical treatments.

Previous studies have showed it is not uncommon to experience inadequate fixation following allograft implantation [12]-[14]. This is due to an inability to immobilize the animal post operatively. Although our results

Table 4. (a) Simple score by rater overall. (b) Percent score by rater overall.

(a)

\begin{tabular}{cccc}
\hline & & Simple Score & \\
Rater & $\mathbf{0}$ & 2.5 & 3.0 \\
\hline Rater 1 & $1(20 \%)$ & $2(40 \%)$ & $2(40 \%)$ \\
Rater 2 & $1(20 \%)$ & $2(40 \%)$ & $2(40 \%)$ \\
Rater 3 & $1(20 \%)$ & $2(40 \%)$ & $2(40 \%)$ \\
\hline
\end{tabular}

$\left(\chi^{2}=1, \mathrm{p}=1.0\right)$.

(b)

\begin{tabular}{cccc}
\hline Rater & & \% Score & range \\
\cline { 2 - 4 } Rater 1 & $\mathbf{n}$ & mean & $(16.6-100)$ \\
Rater 2 & 5 & 78.56 & $(16.6-100)$ \\
Rater 3 & 5 & 78.56 & $(16.6-100)$ \\
\hline
\end{tabular}

$(Z=1, p=1.0)$.

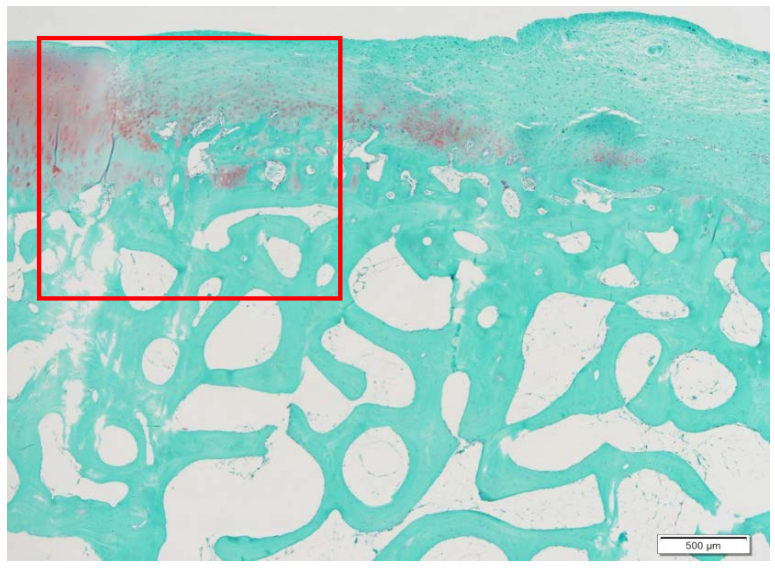

(a)



(b)

Figure 4. (a) 4X Magnification, Safranin-O Stain of HSAM sample. (b) 20X Magnification, Safranin-O Stain of HSAM sample. Proteogly can production at the base of the defect. 


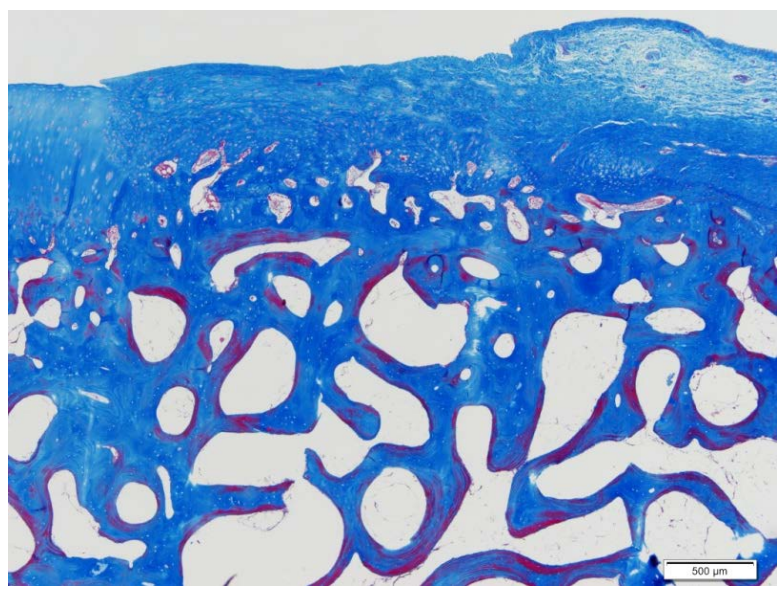

(a)

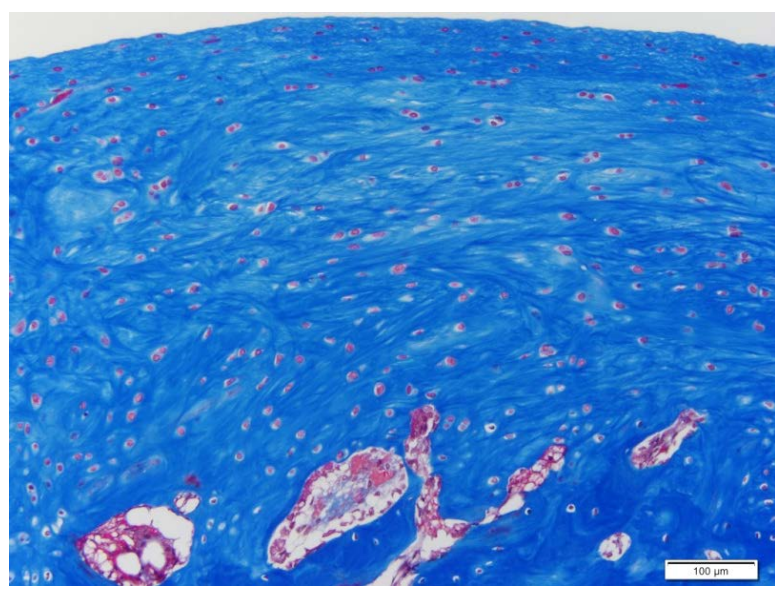

(b)

Figure 5. (a) 4X Magnification, Masson's Trichrome Stain of HSAM sample. (b) 20X Magnification, Masson’s Trichrome Stain of HSAM sample.

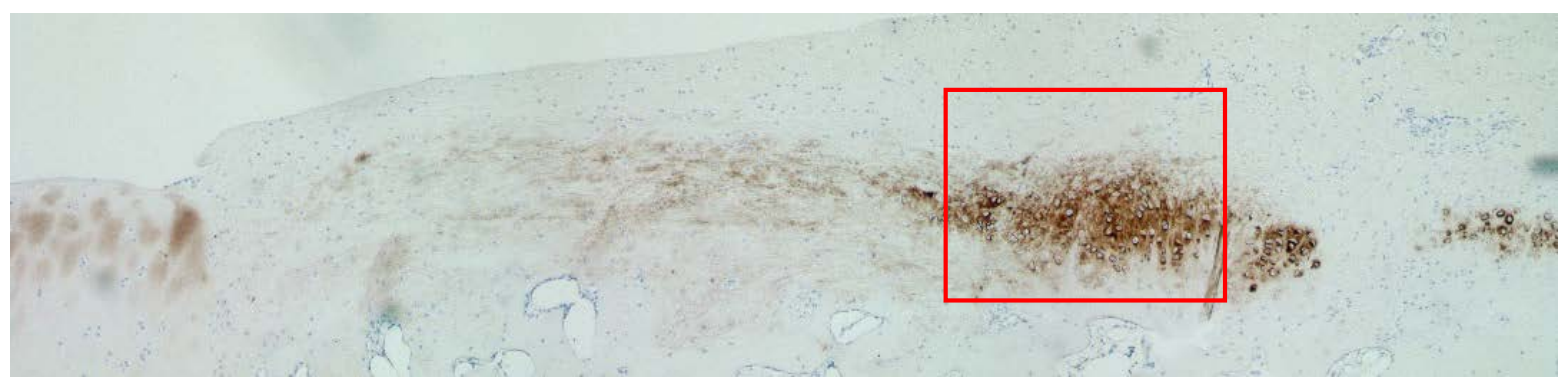

(a)

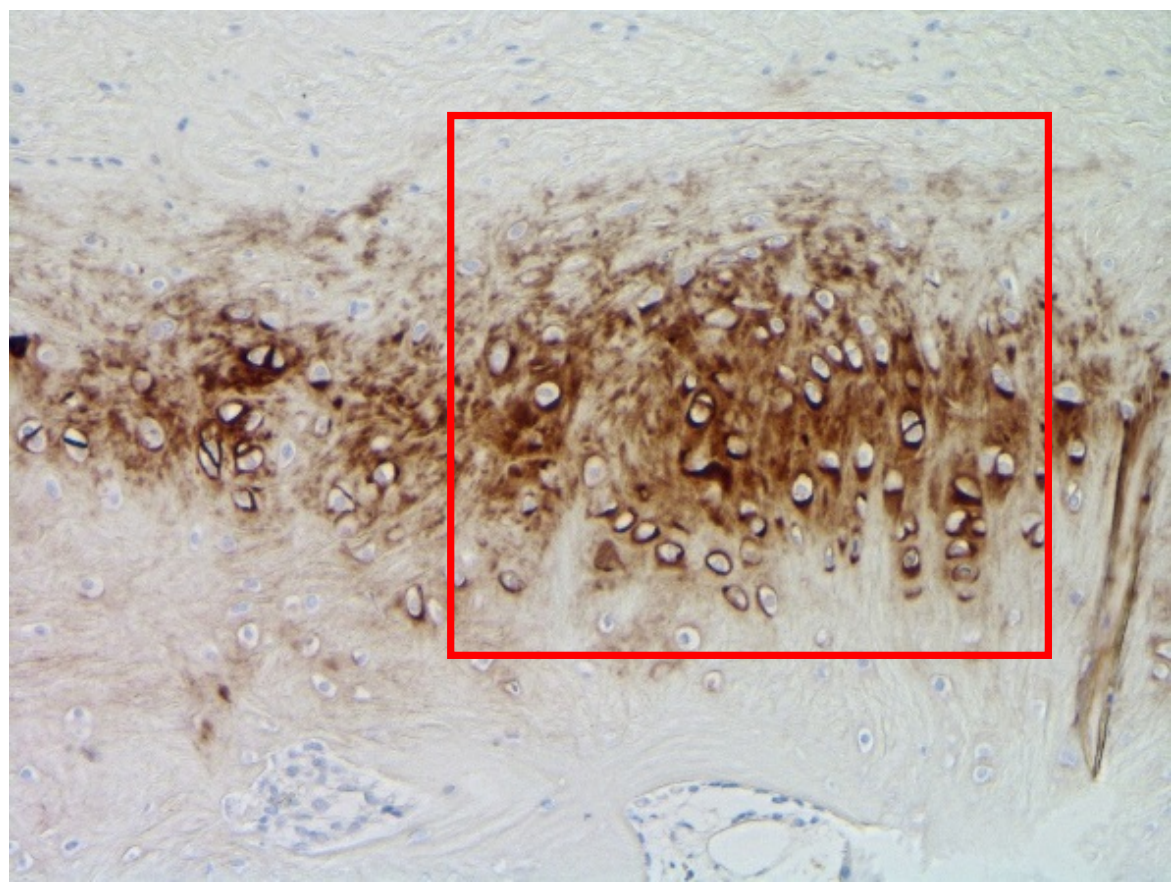

(b)

Figure 6. (a) 10X Magnification of HSAM: Collagen II. (b) 20X Magnification of HSAM: Collagen Type II. Type II collagen formation at the base of the defect. 


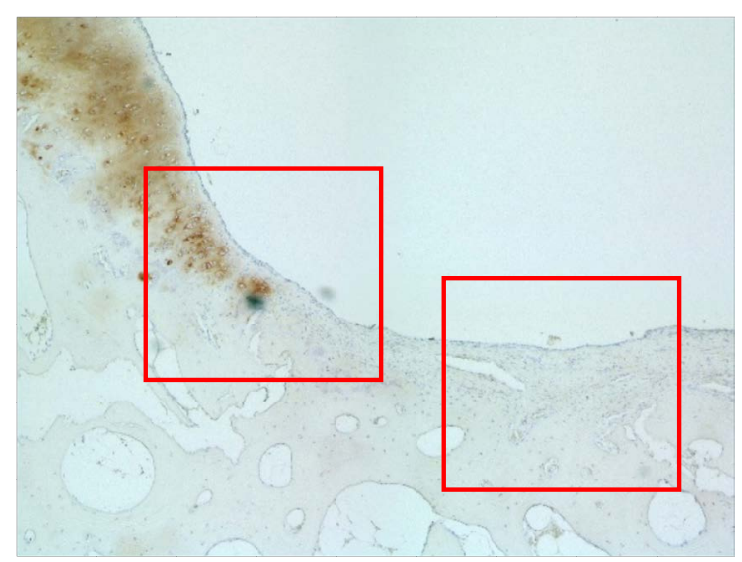

(a)



(b)

Figure 7. (a) 10X Magnificationof control sample: No Collagen II. (b) 20X Magnification of control sample: No Collagen Type II. No type II collagen formation at the base of the defect in the control.

were more limited in scope than we had hoped, a loss of $50 \%$ of the grafts in this study was not surprising. In this study, a decision was made by the licensed veterinarian to not immobilize the sheep due to unnecessary risk it would cause for the animals. Use of a larger sample size would be ideal since immobilization is difficult.

Observing the histological stains of the samples, all three raters rated the control, normal and HSAM graft samples the same in simple score and percent score respectively. The retained HSAM grafts each scored 2.5 on a 0 - 3 cartilage appearance scale compared with 0.5 for the control defects. As the three raters determined, with agreement, the HSAM grafts appeared to be specifically of the mixed hyaline type of cartilage. Safranin-O staining revealed a presence of proteoglycan productivity in the HSAM defects which further indicated the produced matrix was closer to hyaline-like cartilage. Integration of the subchondral bone and the new cartilage was seen in the HSAM graft samples upon Masson’s Trichrome Staining. Further, immunohistochemistry revealed Type II Collagen present in the HSAM graft sample, further indicating a basis for hyaline-like cartilage. Type II collagen was not found in the control sample. These combined results, along with the consistency and reliability of the independent raters are a strong indicator that HSAM has the potential to be influenced to produce hyaline-like cartilage.

This study promotes promising results; hypothermically stored amniotic membrane is a potential source of pluripotent cells that can be influenced to produce specifically hyaline-like cartilage in a sheep model. The implications for application in a human model are promising. The expectations are realistic, as many studies already have shown the potential uses of human amniotic cells in various platforms of tissue repair [3]-[8]. Harnessing the pluripotency of human amniotic cells to produce a stroma rich in chondrocytes and much like normal hyaline cartilage is potentially the next step in providing surgeons with a greater number of options for cartilage restoration.

Weaknesses in this study include the small sample size and the lack of immobilization of the sheep. Future studies should increase the sample size in order to incorporate greater statistical power with which to make deductions and to account for potential of graft loss. Alternatively, the ability to immobilize the sheep would decrease the potential of graft loss, thereby increasing the sample size. Incorporating these changes will lend greater clarity on the already promising concept of using HSAM alone to create hyaline cartilage in sheep models and eventually human patients.

\section{Acknowledgements}

We would like to thank Dr. Douglas Thal for assisting with procuring the sheep for this study and ensuring the ethical treatment of these animals. Additionally, we appreciate NuCel Inc. for funding this study and New Mexico Orthopaedics Associates for allowing lab space for experimental procedures associated with this study.

\section{References}

[1] Moran, C.J., Pascual-Garrido, C., Chubinskaya, S., Potter, H.G., Warren, R.F., Cole, B.J. and Rodeo, S.A. (2014) Res- 
toration of Articular Cartilage. The Journal of Bone \& Joint Surgery, 96, 336-344. http://dx.doi.org/10.2106/jbjs.l.01329

[2] Falah, M., Nierenber, G., Soudry, M., Hayden, M. and Volpin, G. (2010) Treatment of Articular Cartilage Lesions of the Knee. International Orthopaedocs, 34, 621-630. http://dx.doi.org/10.1007/s00264-010-0959-y

[3] Boo, L., Sofiah, S., Selvaratnam, L., Tai, C., Pingguan-Murphy, B. and Kamarul, T. (2009) A Preliminary Study of Human Amniotic Membrane as a Potential Chondrocyte Carrier. Malaysian Orthopaedic Journal, 3, 16-23. http://dx.doi.org/10.5704/MOJ.0911.004

[4] Akle, C.A., Adinolfi, M., Welsh, K.I., Leibowitz, S. and McColl, I. (1981) Immunogenicity of Human Amniotic Epithelial Cells after Transplantation into Volunteers. The Lancet, 2, 1003-1005. http://dx.doi.org/10.1016/S0140-6736(81)91212-5

[5] He, H., Li, W., Tseng, D., Zhang, S., Chen, S., Day, A. and Tseng, S. (2009) Biochemical Characterization and Function of Complexes Formed by Hyaluronan and the Heavy Chains of Inter- $\alpha$-inhibitor (HC-HA) Purified from Extracts of Human Amniotic Membrane. The Journal of Biological Chemistry, 284, 20136-20146. http://dx.doi.org/10.1074/jbc.M109.021881

[6] Marongiu, F., Gramignoli, R., Sun, Q., Tahan, V., Miki, T., Dorko, K., Ellis, E. and Strom, S.C. (2010) Isolation of Amniotic Mesenchymal Stem Cells. Current Protocols in Stem Cell Biology, 12, 1E5.1-1E5.11. http://dx.doi.org/10.1002/9780470151808.sc01e05s12

[7] Jin, C.Z., Park, S.R., Choi, B.H., Lee, K., Kang, C.K. and Min, B.H. (2007) Human Amniotic Membrane as a Delivery Matric for Articular Repair. Tissue Engineering, 14, 693-702. http://dx.doi.org/10.1089/ten.2006.0184

[8] Garcia, Longo, Vaquero, Forriol, Loppini, Khan and Denaro (2015) Amniotic Membrane Transplant for Articular Cartilage Repair: An experimental Study in Sheep. Current Stem Cell Research and Therapy, 10, 77-83.

[9] Diaz-Prado, S., Rendal-Vazquez, M.E., Muines-Lopez, E., Hermida-Gómez, T., Rodríguez-Cabarcos, M., FuentesBoquete, I., et al. (2010) Potential Use of the Human Amniotic Membrane as Scaffold in Human Articular Cartilage Repair. Cell and Tissue Banking, 11, 183-195. http://dx.doi.org/10.1007/s10561-009-9144-1

[10] Hao, Y., Ma, D.H., Hwang, D.G., Kim, W.S. and Zhang, F. (2000) Identification of Antiangiogenic and Antiinflammatory Proteins in Human Amniotic Membrane. Cornea, 19, 348-352. http://dx.doi.org/10.1097/00003226-200005000-00018

[11] O’Driscoll, S.W., Marx, R.G., Beaton, D.E., Miura, Y., Gallay, S.H. and Fitzsimmons, S.H. (2002) Validation of a Simple Histological-Histochemical Cartilage Scoring System. Tissue Engineering, 7, 313-320. http://dx.doi.org/10.1089/10763270152044170

[12] Bentley (1992) Articular Tissue Grafts. Annals of Rheumatic Diseases, 51, 292-296. http://dx.doi.org/10.1136/ard.51.3.292

[13] Driesand, I.M. and Hunziker, E.B. (2000) Delamination Rates of Tissue Flaps Used in Articular Cartilage Repair. Journal of Orthopaedic Research, 18, 909-911. http://dx.doi.org/10.1002/jor.1100180609

[14] Seedhom, B.B., Luo, Z.-J., Goldsmith, A.J., Toyoda, T., Lorrison, J.C. and Guardamagna, L. (2007) In-Situ Engineering of Cartilage Repair: A Pre-Clinical in-Vivo Exploration of a Novel System. Journal of Engineering in Medicine, 221, 475-487. http://dx.doi.org/10.1243/09544119JEIM188 